\title{
Patient-Reported Outcome Measures May Add Value in Breast Cancer Surgery
}

\author{
M. Lagendijk, MD ${ }^{1}$, L. S. E. van Egdom, MD ${ }^{1}$, F. E. E. van Veen ${ }^{1}$, E. L. Vos, MD, MSc ${ }^{1,2}$, M. A. M. Mureau, MD, \\ $\mathrm{PhD}^{3}$, N. van Leeuwen, $\mathrm{MSc}^{2}$, J. A. Hazelzet, MD, $\mathrm{PhD}^{2}$, H. F. Lingsma, PhD, MSc ${ }^{2}$, and L. B. Koppert, MD, PhD, \\ MSc $^{1}$
}

${ }^{1}$ Department of Surgery, Erasmus MC, Rotterdam, The Netherlands; ${ }^{2}$ Department of Public Health, Erasmus MC, Rotterdam, The Netherlands; ${ }^{3}$ Department of Plastic and Reconstructive Surgery, Erasmus MC Cancer Institute,

Rotterdam, The Netherlands

\begin{abstract}
Purpose. Considering the comparable prognosis in earlystage breast cancer after breast-conserving therapy (BCT) and mastectomy, quality of life should be a focus in treatment decision(s). We retrospectively collected PROs and analyzed differences per type of surgery delivered. We aimed to obtain reference values helpful in shared decisionmaking.

Patients and Methods. pTis-T3N0-3M0 patients operated between January 2005 and September 2016 were eligible if: (1) no chemotherapy was administered $<6$ months prior to enrolment, and (2) identical surgeries were performed in case of bilateral surgery. After consent, EQ-5D-5L, EORTC-QLQ-C30/BR23, and BREAST-Q were administered. PROs were evaluated per baseline characteristics using multivariable linear regression models. Outcomes were compared for different surgeries as well as for primary (PBC) and second primary or recurrent (SBC) breast cancer patients using analyses of variance (ANOVAs).

Results. The response rate was $68 \%$. PROs in 612 PBC patients were comparable to those in 152 SBC patients. Multivariable analyses showed increasing age to be associated with lower "physical functioning" $[\beta-0.259$,
\end{abstract}

Electronic supplementary material The online version of this article (https://doi.org/10.1245/s10434-018-6729-6) contains supplementary material, which is available to authorized users.

(C) The Author(s) 2018

First Received: 13 April 2018

M. Lagendijk, MD

e-mail: mirellelagendijk@gmail.com

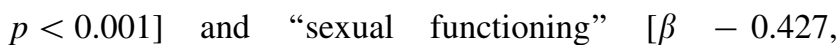
$p<0.001]$, and increasing time since surgery with less "fatigue" $[\beta-1.083, \quad p<0.001]$. Mastectomy $[\beta$ $-13.596, \quad p=0.003]$ and implant reconstruction $[\beta$ $-13.040, p=0.007]$ were associated with lower "satisfaction with breast" scores than BCT. Radiation therapy was associated with lower satisfaction scores than absence of radiotherapy.

Discussion. PRO scores were associated with age, time since surgery, type of surgery, and radiation therapy in breast cancer patients. The scores serve as a reference value for different types of surgery in the study population and enable prospective use of PROs in shared decisionmaking.

Breast cancer is the most frequently diagnosed cancer in women. ${ }^{1}$ In The Netherlands, 1 in 7 women are diagnosed with breast cancer. ${ }^{2}$ Favorable high survival rates are reported eminently in early stages. ${ }^{3}$ Survivorship as well as physical, sexual, and psychosocial consequences of breast cancer therapies should therefore be accounted for in treatment decision-making. In early-stage breast cancer, high survival rates are achieved irrespective of type of surgery, whether breast-conserving therapy (BCT; breastconserving surgery with breast radiation therapy) or mastectomy (with or without reconstruction). ${ }^{4-6}$ Consequently, anticipation of outcomes reflecting physical, sexual, and psychosocial functioning is very important in treatment decision-making in these patients.

Value in healthcare is defined as the health outcome per total cost. Multiple health outcomes are often used for one medical condition to define this value. ${ }^{7}$ In value-based healthcare (VBHC), the defined outcomes are patient 
oriented and therefore form a combination of more traditional clinical outcomes (for example, oncological outcome or complication rates) and patient-reported outcomes (PROs). Collaborations of the International Consortium for Health Outcomes Measurement (ICHOM) with several other healthcare institutions worldwide have resulted in the development of a standard breast cancer outcome set. ${ }^{8}$ Incorporation of this set is expected to pave the way towards value-based breast cancer care with application in shared decision-making as well as follow-up.

Patient-reported outcome measures (PROMs) are pivotal in the ICHOM breast cancer outcome set, accounting for approximately $75 \%$ of outcomes, the other $25 \%$ being related to clinical outcomes. Little is known about PROM scores following different surgeries in relation to differences in patient, tumor, and systemic or radiation treatment characteristics. Our institute implemented a breast cancer outcome set embedded in the institutional VBHC initiative in October 2015. At predetermined time points, breast cancer patients received digitalized PRO questionnaires prior to their routine visit at the outpatient clinic. PROs were evaluated with the patient at the outpatient clinic and used to improve individual care. ${ }^{9}$ Consequently, there was an urgent need to propose valid and meaningful reference scores. It was hypothesized that PROs differ between surgical treatments. The aim of this study is to assess the correlation between PROs and patient, tumor, and treatment characteristics and to provide PRO reference values for different breast cancer surgeries. We therefore collected PROs amongst breast cancer patients operated in the last 10 years within our institute.

\section{PATIENTS AND METHODS}

\section{Study Population}

Ethical approval was granted by the Institutional Review Board of the Erasmus Medical Centre (Erasmus MC), Rotterdam, The Netherlands (MEC-2015-669). Patients who had undergone breast cancer surgery between January 2005 and September 2016 were identified from electronic patient files using operation codes. Women aged $>18$ years with pTis-3N0-3M0 breast cancer were deemed eligible. Patients were excluded if they had been treated with chemotherapy within 6 months prior to the PRO assessment or had bilateral breast surgery with different types of surgery performed per side.

\section{Procedures}

This cross-sectional study retrospectively reviewed medical records to compile the following data: age, date and type of breast surgery, tumor morphology, tumornode-metastasis (TNM) staging according to the TNM classification system (7th edition), ${ }^{10}$ hormonal receptor status, human epidermal growth factor receptor 2 (HER2) status, BRCA1/2 status, local recurrence, second primary breast cancer, and details regarding chemotherapy and/or immunotherapy and endocrine therapy. Time since surgery was defined as time between first surgery and questionnaire completion. The respondents were categorized into those with "primary breast cancer" (PBC) and "second primary or recurrent breast cancer" (SBC). PBC patients represented women with primary unilateral or bilateral breast cancer, while SBC patients represented women with local recurrence or second primary breast cancer. In case of breast cancer recurrence or second primary breast cancer, data regarding patient age, tumor morphology, and TNM stage of the primary diagnosis was used.

Operation types defined were: breast-conserving therapy (BCT), mastectomy alone (MAS), mastectomy followed by immediate or delayed implant reconstruction (REC I), and mastectomy followed by immediate or delayed autologous reconstruction (REC A). Nodal stage at primary diagnosis was categorized as $N 0, N+(N 1-3)$, or unknown. Adjuvant systemic therapy was categorized as: (1) no systemic therapy, (2) chemotherapy and/or immunotherapy (CTx), (3) endocrine therapy (ETx), (4) chemotherapy/immunotherapy and endocrine therapy (CTx \& ETx), or (5) unknown. Radiotherapy was categorized as (1) radiation therapy following breast-conserving surgery, (2) no radiation therapy, or (3) thoracic wall radiotherapy in case of mastectomy and/or locoregional radiotherapy in case of mastectomy or BCT.

Eligible women were contacted by telephone to request their participation. Upon oral informed consent, details on adjuvant therapy and last breast surgery were verified. Patients who did not answer were called up to six times, after which participation was no longer pursued.

Following consent, four questionnaires were administered; Euro-QoL 5D-5L (EQ-5D-5L version 2.0). ${ }^{11}$ The European Organization of Research and Treatment of Cancer quality of life questionnaires (EORTC-QLQ-C30 version $3.0^{12}$ and EORTC-QLQ-BR23 version $1.0^{13}$ ), and BREAST-Q (postoperative version 1.0). ${ }^{14}$ The questionnaires used are proposed in the ICHOM breast cancer outcome set to evaluate breast cancer patients undergoing locoregional treatment(s). ${ }^{8}$ The EORTC-QLQ-C30 is a generic oncologic questionnaire containing 30 questions with 6 single-item scores, 9 multiitem scales, 3 symptom scales, and an additional global health status/quality of life (QoL) scale. ${ }^{15}$ The EORTC-QLQ-BR23 is a breast cancerspecific questionnaire of the EORTC QLG that contains 23 questions made up of 8 multiple-item scales and is considered an addition to the EORTC-QLQ-C30 specifically 
for breast cancer patients. The BREAST-Q is a surgeryspecific questionnaire proposed in the ICHOM set to measure "satisfaction with breast" following breast cancer surgery. Multiitem domains are, however, also available to evaluate "satisfaction with overall outcome," "psychosocial well-being," "sexual well-being," "physical wellbeing," and "satisfaction with care." "In the current study, all modules of the BREAST-Q except "satisfaction with overall outcome" were used.

Patients were given the choice of internet-based questionnaires sent by email or paper-based questionnaires sent by mail (with postage-paid return envelope). If the questionnaires remained uncompleted, a weekly reminder up to 3 weeks was sent by email (internet-based). After 4 weeks of no response, patients were contacted by telephone and requested to complete questionnaires (internet-based and paper-based). Thereafter, response was no longer actively pursued. PRO scores were calculated according to questionnaire scoring manual. PROs were evaluated for patients who completed at least the EORTC-QLQ-C30 questionnaire.

\section{Study Outcomes}

The primary aim was to obtain reference values for PROs following different surgical strategies in relation to patient, tumor, and treatment characteristics of $\mathrm{PBC}$ patients. Additionally, PROs of SBC patients were evaluated and compared with PROs of PBC patients.

\section{Statistical Analysis}

All analyses were performed using SPSS Statistics for Windows (version 21.0). Baseline characteristics were compared for responders versus nonresponders and $\mathrm{PBC}$ versus SBC patients. The different surgical groups were compared within both $\mathrm{PBC}$ and SBC using one-way ANOVA. Post hoc analyses were performed to detect differences between specific groups. To evaluate the effect of patient, tumor, and treatment on PROs, a multivariate linear regression was used in PBC patients. Factors evaluated were age, time since surgery, uni/bilateral breast cancer, $B R C A$ mutation status, tumor stage, nodal stage, systemic therapy status, and radiotherapy status. Beta coefficients $(\beta)$ with corresponding $p$-values were calculated for the index value (EQ-5D-5L), "global health status"/"physical functioning"/"role functioning" (EORTC-QLQ-C30), "body image"/"sexual functioning" (EORTC-QLQBR23), and "Q-satisfaction with breast"/"Q-physical"/ "Q-psychosocial," and "Q-sexual" (BREAST-Q). $p$ values $\leq 0.01$ were considered statistically significant.

\section{RESULTS}

Out of 1850 patients identified, 1230 (66.5\%) had pTisT3N0-3M0 breast cancer at primary diagnosis. A total of 1116 (90.7\%) were eligible for participation (Fig. 1). Of the eligible patients, $764(68.5 \%)$ responded. Of 352 nonresponders, $114(40.9 \%)$ could not be reached, $162(46 \%)$ declined participation, and $46(13.1 \%)$ did not complete the EORTC-QLQ-C30.

\section{Study Population}

Responders Versus Nonresponders Responders were significantly younger compared with nonresponders (50.5 vs 52.4 years, $p=0.04$ ). Significant differences were additionally found for type of surgery performed, $\mathrm{T}$ stage, and systemic and radiation therapy (Supplementary Table S1).

Responders: Baseline Characteristics and Treatment A total of $612(80.1 \%)$ responders had PBC (Table 1), and 152 (19.9\%) women had SBC (Supplementary Table S2). Of PBC patients, 257 (41.9\%) underwent BCT, 162 (26.6\%) mastectomy, 110 (17.9\%) implant reconstruction, and $83(13.5 \%)$ autologous reconstruction (Table 1). PBC patients showed significant differences between the surgical groups for age, time since surgery, unilateral/bilateral surgery, $\mathrm{T}$-stage, $\mathrm{N}$-stage, systemic therapy, radiation therapy, and $B R C A$ mutation status (Table 1).

\section{Patient-Reported Outcomes}

Completion rates for the individual PRO modules in respondents ranged between 88 and $100 \%$, with the exception of the Q-sexual module, which showed lower response rates (Table 2, Supplementary Table S3). Statistically significant differences between surgical treatments were found in the PBC group in "physical functioning," "sexual functioning,", and all Q-scores on univariate analyses (Table 2). Post hoc analyses showed that mastectomy patients overall reported significantly lower mean scores on "physical functioning" (80.1) compared with BCT $(86.4, p=0.001)$, compared with implant (92.6, $p<0.001)$, and compared with autologous reconstruction (87.5, $p=0.006)$. "Body image" was lower following mastectomy (75.7) compared with BCT (83.9, $p=0.005)$. Significantly lower "sexual functioning" scores (EORTCQLQ-BR23) were reported by BCT patients (24.2) compared with implant $(36.6, p<0.001)$ and autologous reconstruction (33.6, $p=0.001$ ) patients. Lower mean "sexual functioning" scores were also reported by mastectomy patients (20.6) compared with both implant and 
FIG. 1 Flowchart of study selection process. $T$ tumor stage, $N$ nodal stage, $P B C$ primary breast cancer, $S B C$ second primary or recurrent breast cancer

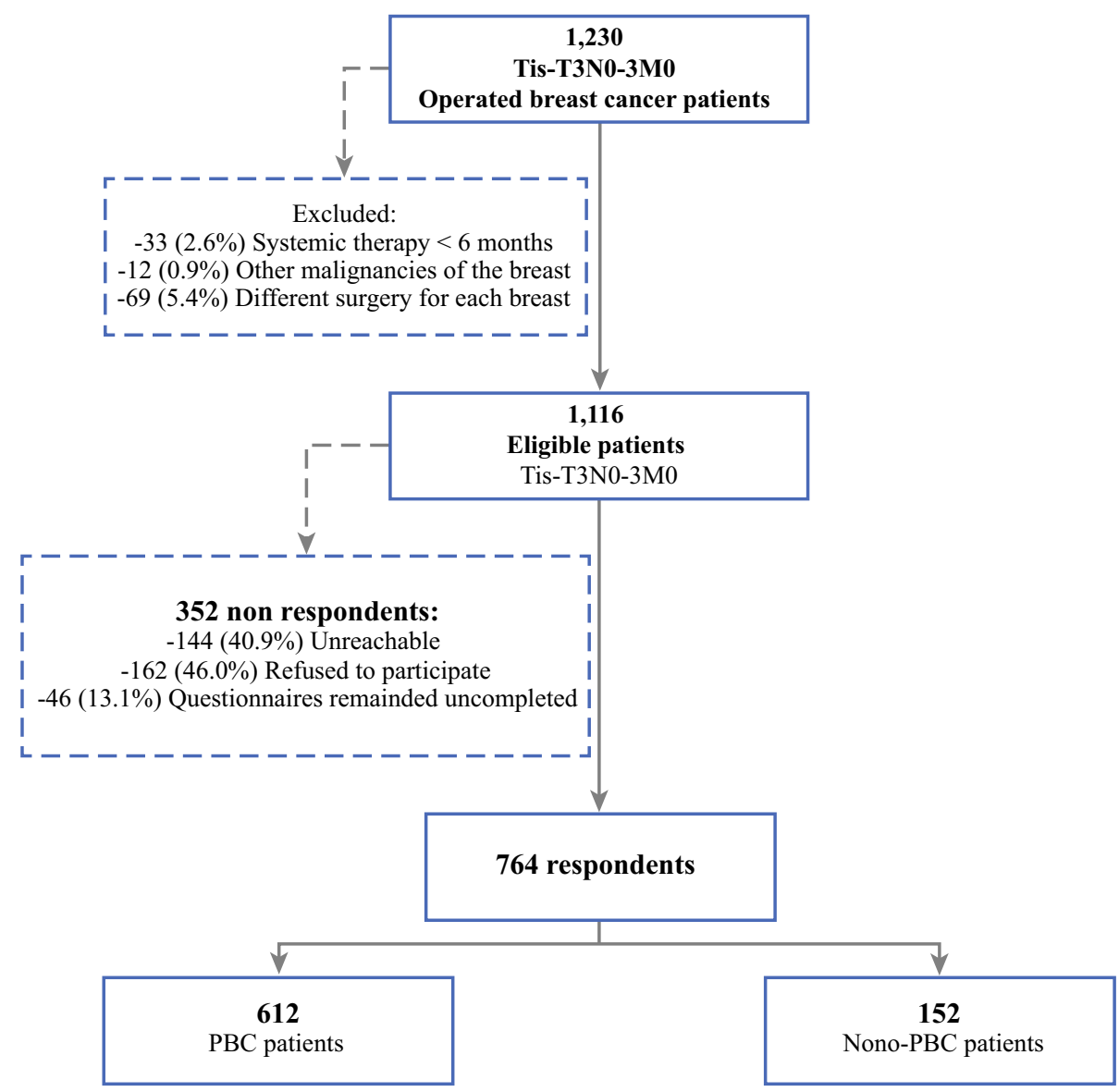

autologous reconstruction patients $(p<0.001$ and $p=0.001$, respectively). "Q-psychosocial" was lower following mastectomy (65.8) compared with implant (74.1, $p=0.004)$ and autologous reconstruction (75.7, $p<0.001)$. Mean "Q-satisfaction with breast" reported by mastectomy patients (61.7) was significantly lower compared with BCT $(65.7, p=0.006)$ and autologous reconstruction patients $(70.5, p=0.004)$. No significant differences in outcome were found between the different surgeries in the SBC group (data not shown).

When evaluating PROs in multivariate analyses, increasing age was related to lower scores on "physical functioning" $(\beta-0.259, p<0.001)$ and "sexual functioning" $(\beta-0.427, p<0.001)$ (Table 3$)$. Longer time since surgery was associated with less "fatigue" $(\beta$ - 1.083, $p<0.001$ ) (Supplementary Table S4). "Q-satisfaction with breast" was significantly lower for patients following mastectomy $(\beta-13.596, p=0.003)$ and implant reconstruction $(\beta-13.040, p=0.007)$ compared with BCT (Table 3 ). If patients had not undergone radiation therapy, "Q-satisfaction with breast" was significantly better than following BCT (with consequent radiation therapy) ( $\beta$ 11.956, $p<0.009$ ) (Table 3).

\section{DISCUSSION}

Breast cancer patients are faced with complex treatment decision(s) shortly after breast cancer diagnosis. Insights into not only prognosis but also quality of life or daily functioning resulting from these decisions could improve the shared decision-making process and ultimately the care delivered. Knowledge on QoL related to different surgical treatments is urgently needed. The aim of this study is to obtain and evaluate PROs in breast cancer patients according to the surgery performed. Collected PROs indeed showed statistically significant differences for the various surgeries performed. The collected PROs can serve as reference and ultimately pave the way for implementation of value-based healthcare among future breast cancer patients at the outpatient clinic.

In primary breast cancer patients, PRO scores in mastectomy patients were lower compared with $\mathrm{BCT}$ or breast reconstruction patients, except for "Q-physical" and "Qsatisfaction with breast." Both mastectomy patients and patients with implant reconstruction reported significantly lower "satisfaction with breast" scores compared with BCT or autologous reconstruction patients. These results corroborate previous studies which showed lower 
TABLE 1 Baseline characteristics of 612 primary breast cancer patients per type of surgery, $\mathrm{n}(\%)$

\begin{tabular}{|c|c|c|c|c|c|c|}
\hline & All $n=612$ & BCT $n=257$ & MAS $n=162$ & REC I $n=110$ & REC A $n=83$ & $p$ value ${ }^{\S}$ \\
\hline Median (IQR) age (years) & $51.0(43.0-60.0)$ & $54.0(48.0-62.0)$ & $55.0(47.0-63.0)$ & $42.5(36.0-51.0)$ & $45.0(37.0-52.0)$ & $<0.001^{¥}$ \\
\hline Median (IQR) time since surgery (years) & $6.3(3.3-9.4)$ & $5.3(2.8-8.1)$ & $7.1(3.7-9.8)$ & $7.0(3.7-10.4)$ & $7.2(4.7-9.5)$ & $<0.001^{¥}$ \\
\hline Surgery & & & & & & $<0.001$ \\
\hline Unilateral & $475(77.6)$ & $251(97.7)$ & $133(82.1)$ & $51(46.4)$ & $40(48.2)$ & \\
\hline Bilateral & $137(22.4)$ & $6(2.3)$ & 29 (17.9) & $59(53.6)$ & $43(51.8)$ & \\
\hline Unknown & .0 & .0 & .0 & .0 & .0 & \\
\hline T stage & & & & & & $<0.001$ \\
\hline $\mathrm{T} 1$ & $354(57.8)$ & $173(67.3)$ & $86(53.1)$ & $58(52.7)$ & $39(47.0)$ & \\
\hline $\mathrm{T} 2$ & $128(20.9)$ & $42(16.3)$ & $51(31.5)$ & $14(12.7)$ & $21(25.3)$ & \\
\hline $\mathrm{T} 3$ & $18(2.9)$ & .0 & $8(4.9)$ & $4(3.6)$ & $6(7.2)$ & \\
\hline CIS & $108(18.0)$ & $42(16.3)$ & $17(10.5)$ & $33(30.0)$ & $16(19.3)$ & \\
\hline Unknown & $2(0.3)$ & .0 & .0 & $1(0.9)$ & $1(1.2)$ & \\
\hline $\mathrm{N}$ stage & & & & & & $<0.001$ \\
\hline N0 & $442(72.2)$ & $211(82.1)$ & $91(56.2)$ & $90(81.8)$ & $50(60.2)$ & \\
\hline $\mathrm{N}+$ & $170(27.8)$ & $46(17.9)$ & $71(43.8)$ & $20(18.2)$ & $33(39.8)$ & \\
\hline Unknown & .0 & .0 & .0 & .0 & .0 & \\
\hline Systemic therapy & & & & & & $<0.001$ \\
\hline None & $256(41.8)$ & $130(50.6)$ & $46(28.4)$ & $53(48.2)$ & $27(32.5)$ & \\
\hline CTx & $88(14.4)$ & $26(10.1)$ & $20(12.3)$ & $16(14.5)$ & $25(30.1)$ & \\
\hline ETx & $95(15.5)$ & $49(19.1)$ & $34(21.0)$ & $10(9.1)$ & $2(2.4)$ & \\
\hline CTx \& ETx & $173(28.3)$ & $52(20.2)$ & $62(38.3)$ & $31(28.2)$ & 29 (34.9) & \\
\hline Unknown & .0 & .0 & .0 & .0 & .0 & \\
\hline Radiation therapy & & & & & & $<0.001$ \\
\hline RTx following BCS & $233(38.1)$ & $233(90.7)$ & .0 & .0 & .0 & \\
\hline No RTx & $293(47.9)$ & $14(5.4)$ & $120(74.1)$ & $94(85.5)$ & $65(78.3)$ & \\
\hline Thoracic-wall and/or locoregional RTx & 85 (13.9) & $9(3.5)$ & $42(25.9)$ & $16(14.5)$ & $18(21.7)$ & \\
\hline Unknown & $1(0.2)$ & $1(0.4)$ & .0 & .0 & .0 & \\
\hline$B R C A$ & & & & & & $<0.001$ \\
\hline$B R C A 1 / 2$ negative & $399(65.2)$ & $179(69.6)$ & $113(69.8)$ & $58(52.7)$ & $49(59.0)$ & \\
\hline$B R C A 1 / 2$ positive & $90(14.7)$ & $13(5.1)$ & $15(9.3)$ & $36(32.7)$ & $26(31.3)$ & \\
\hline Unknown & $123(20.1)$ & $65(25.3)$ & $34(20.9)$ & $16(14.6)$ & $8(9.6)$ & \\
\hline Histological type & & & & & & 0.003 \\
\hline IDC & $415(67.8)$ & $185(72.0)$ & $113(69.8)$ & $58(52.7)$ & $59(71.1)$ & \\
\hline ILC & $44(7.2)$ & $16(6.2)$ & $16(9.9)$ & $7(6.4)$ & $4(4.8)$ & \\
\hline CIS & $111(18.2)$ & $41(16.0)$ & 19 (11.7) & $35(31.8)$ & $16(19.3)$ & \\
\hline Other & $35(5.7)$ & $15(5.8)$ & $12(7.4)$ & $7(6.4)$ & $2(2.4)$ & \\
\hline Unknown & $7(1.1)$ & .0 & $2(1.2)$ & $3(2.7)$ & $2(2.4)$ & \\
\hline Differentiation grade ${ }^{\wedge}$ & & & & & & $<0.001$ \\
\hline Grade 1 & $100(16.3)$ & $61(23.7)$ & $21(13.0)$ & $11(10.0)$ & $7(8.4)$ & \\
\hline Grade 2 & $216(35.3)$ & $100(38.9)$ & $63(38.9)$ & $31(28.2)$ & $20(24.1)$ & \\
\hline Grade 3 & $160(26.1)$ & $49(19.1)$ & $55(34.0)$ & $24(21.8)$ & $32(38.6)$ & \\
\hline NA & $113(18.5)$ & $44(17.1)$ & 19 (11.7) & $35(31.8)$ & $16(19.3)$ & \\
\hline Unknown & $23(3.8)$ & $3(1.2)$ & $4(2.5)$ & $9(8.2)$ & $8(9.6)$ & \\
\hline
\end{tabular}

$B C T$ breast-conserving therapy, MAS mastectomy, REC I mastectomy followed by (in)direct implant reconstruction, REC A mastectomy followed by (in)direct autologous reconstruction, $C T x$ chemotherapy and/or immunotherapy, ETx endocrine therapy, $R T x$ radiation therapy, IDC invasive ductal carcinoma, ILC invasive lobular carcinoma, CIS carcinoma in situ

${ }^{\S}$ Chi squared test

${ }^{¥}$ Kruskall-Wallis test 
TABLE 2 Mean (SD) PRO scores per type of surgery in 612 primary breast cancer patients, n (\%)

\begin{tabular}{|c|c|c|c|c|c|}
\hline & $\mathrm{BCT}(n=257)$ & MAS $(n=162)$ & $\operatorname{REC} \mathrm{I}(n=110)$ & $\operatorname{REC} \mathrm{A}(n=83)$ & $p$ value $^{¥}$ \\
\hline \multicolumn{6}{|l|}{ EQ-5D-5L } \\
\hline Index value & $0.83(0.14)[93]$ & $0.81(0.16)$ [96] & $0.86(0.15)[98]$ & $0.85(0.14)$ & 0.036 \\
\hline \multicolumn{6}{|l|}{ EORTC-QLQ-C30 } \\
\hline Global health status & $79.7(17.5)$ & $76.2(19.5)$ & $82.6(18.6)$ & $81.3(17.5)$ & 0.026 \\
\hline Physical function & $86.4(14.4)$ & $80.1(19.6)$ & $92.6(9.8)$ & $87.5(14.6)$ & $<0.001 *$ \\
\hline Role function & $85.0(21.3)$ & $78.0(26.0)$ & $86.2(22.7)$ & $83.7(24.0)$ & 0.01 \\
\hline Fatigue \pm & $25.6(25.0)$ & $28.7(24.1)$ & $21.0(23.0)$ & $24.4(25.3)$ & 0.09 \\
\hline $\operatorname{Pain} \pm$ & $16.6(21.0)$ & $20.9(26.6)$ & $13.2(19.2)$ & $16.1(22.3)$ & 0.042 \\
\hline \multicolumn{6}{|l|}{ EORTC-QLQ-BR23 } \\
\hline Body image $^{\circ}$ & 83.9 (21.3) [99] & 75.7 (26.0) [97] & $77.3(25.1)[90]$ & $81.9(21.0)[95]$ & $0.003 *$ \\
\hline Sexual function $^{\circ}$ & $24.2(20.8)[95]$ & $20.6(22.3)[91]$ & $36.6(24.0)[89]$ & $33.6(24.1)[90]$ & $<0.001^{*}$ \\
\hline \multicolumn{6}{|l|}{ BREAST-Q } \\
\hline Physical well-being & $71.2(18.9)[96]$ & $75.1(19.2)[98]$ & $76.8(10.9)[96]$ & $78.3(13.8)[98]$ & $0.002 *$ \\
\hline Psychosocial well-being & $70.1(21.4)[96]$ & $65.8(18.8)[98]$ & $74.1(20.1)[96]$ & $75.7(17.5)[98]$ & $0.001 *$ \\
\hline Satisfaction with breasts & $65.7(22.4)[95]$ & $61.8(17.7)[96]$ & $61.2(15.7)[96]$ & $70.5(20.2)[98]$ & $0.003 *$ \\
\hline Sexual well-being & $57.5(20.3)[73]$ & $54.7(19.2)[67]$ & $59.3(19.9)[85]$ & $62.4(20.3)[90]$ & 0.07 \\
\hline
\end{tabular}

EQ-5D-5L index-value: scale from - 0.28 to 1.0. EORTC-QLQ-C30/BR23 and BREAST-Q scale 0 to 100

${ }^{\circ}$ Higher scores represent higher quality

\pm Higher scores represent lower quality

[] percentages complete modules if not $100 \%$

¥ANOVA

*Statistically significant differences

satisfaction and impaired sexual, psychosocial, and physical functioning following mastectomy compared with BCT or breast reconstruction. ${ }^{16,17}$ After adjustment for patient, tumor, and treatment characteristics, a significant effect of surgical treatment on "Q-satisfaction with breast" scores persisted. Compared with BCT, statistically significant lower "Q-satisfaction with breast" was reported by mastectomy and implant reconstruction patients. No statistically significant differences were found in PROs between autologous reconstruction and BCT when adjusting for patient, tumor, and treatment characteristics. Contradictory results are found in literature, reporting comparable PRO scores ${ }^{18}$ or scores in favor of autologous reconstruction techniques. "No radiation therapy" was associated with statistically significant higher "Q-satisfaction with breast" scores as compared with BCT patients. Thoracic wall radiation therapy $(25.9,14.5$, and $21.7 \%$ of mastectomy, REC I, and REC A patients, respectively) and locoregional radiotherapy in $3.5 \%$ of the BCT patients was associated with lower Q-satisfaction scores compared with patients who had not undergone radiation therapy. Radiation therapy is therefore an important independent factor for "Q-satisfaction with breast" scores in addition to the type of surgery performed.
Strengths of the current study include the size of the study population and the response rate of $68 \%$. This enabled evaluation of four different PROMs, generating a detailed reflection of quality of life. To the best of the authors' knowledge, this is the largest study to evaluate the complete set of PROs proposed in the ICHOM breast cancer set per type of surgery with adjustment for potential confounders. This is a pivotal step forward towards extensive use of PROMs in clinical research and practice for implementation of VBHC. It furthermore enables future international comparison. When both PROs and baseline characteristics are available, case-mix-corrected comparison between centers can be performed to benchmark.

Limitations include the single-center and retrospective design. Longitudinal PRO collection is proposed when using these outcomes as a clinical tool. Enabling comparison with baseline values is expected to reflect the influence of different treatments better than a single score obtained following treatment. Moreover, not all variables that possibly affect PROs were available for the current cohort, such as socioeconomic status. ${ }^{16}$ Large multicenter initiatives are needed to obtain narrow reference scores as well as the possibility for benchmarking. Evaluation of PROs obtained in retrospect does however generate the necessary insights into factors possibly related to PRO scores. These 
TABLE 3 Multivariate linear regression analyses in 612 primary breast cancer patients

\begin{tabular}{|c|c|c|c|c|c|c|c|c|c|c|c|c|}
\hline & \multicolumn{4}{|c|}{ EORTC-QLQ-C30 } & \multicolumn{4}{|c|}{ EORTC-QLQ-BR23 } & \multicolumn{4}{|c|}{ BREAST-Q } \\
\hline & \multicolumn{2}{|c|}{$\begin{array}{l}\text { Physical } \\
\text { functioning }\end{array}$} & \multicolumn{2}{|c|}{ Body image $^{\circ}$} & \multicolumn{2}{|c|}{ Sexual functioning ${ }^{\circ}$} & \multicolumn{2}{|c|}{ Q-physical $^{\circ}$} & \multicolumn{2}{|c|}{ Q-psychosocial $^{\circ}$} & \multicolumn{2}{|c|}{$\begin{array}{l}\text { Q-satisfaction with } \\
\text { breast }^{\circ}\end{array}$} \\
\hline & $\beta$ & $p$ value & $\beta$ & $p$ value & $\beta$ & $p$ value & $\beta$ & $p$ value & $\beta$ & $p$ value & $\beta$ & $p$ value \\
\hline \multicolumn{13}{|l|}{ Operation type } \\
\hline $\mathrm{BCT}$ & ref & $\mathrm{Na}$ & ref & $\mathrm{Na}$ & ref & $\mathrm{Na}$ & ref & $\mathrm{Na}$ & ref & $\mathrm{Na}$ & ref & $\mathrm{Na}$ \\
\hline MAS & -3.781 & 0.27 & -7.757 & 0.15 & -8.185 & 0.10 & -0.253 & 0.95 & -2.620 & 0.57 & -13.596 & $0.003 *$ \\
\hline REC I & 5.223 & 0.15 & -4.033 & 0.48 & 2.544 & 0.64 & 2.579 & 0.53 & 6.365 & 0.18 & -13.040 & $0.007 *$ \\
\hline REC A & 0.523 & 0.89 & 1.084 & 0.85 & -0.881 & 0.87 & 4.712 & 0.26 & 9.434 & 0.05 & -2.651 & 0.59 \\
\hline Age & -0.259 & $<0.001 *$ & 0.209 & 0.02 & -0.427 & $<0.001^{*}$ & 0.095 & 0.13 & 0.090 & 0.22 & 0.097 & 0.19 \\
\hline $\begin{array}{l}\text { Time since } \\
\text { surgery }\end{array}$ & 0.252 & 0.14 & 0.572 & 0.03 & -0.473 & 0.06 & 0.446 & 0.02 & 0.299 & 0.19 & -0.059 & 0.79 \\
\hline \multicolumn{13}{|l|}{ Surgery } \\
\hline Unilateral & $r e f$ & $\mathrm{Na}$ & $r e f$ & $\mathrm{Na}$ & $r e f$ & $\mathrm{Na}$ & ref & $\mathrm{Na}$ & $r e f$ & $\mathrm{Na}$ & $r e f$ & $\mathrm{Na}$ \\
\hline Bilateral & -3.560 & 0.08 & 1.729 & 0.59 & 2.349 & 0.44 & -3.732 & 0.11 & 2.888 & 0.28 & -0.327 & 0.90 \\
\hline \multicolumn{13}{|l|}{$\mathrm{T}$ stage } \\
\hline $\mathrm{T} 1$ & ref & $\mathrm{Na}$ & ref & $\mathrm{Na}$ & ref & $\mathrm{Na}$ & ref & $\mathrm{Na}$ & ref & $\mathrm{Na}$ & ref & $\mathrm{Na}$ \\
\hline $\mathrm{T} 2$ & 1.481 & 0.36 & -1.757 & 0.51 & -2.595 & 0.31 & 0.1997 & 0.92 & -2.343 & 0.30 & -2.122 & 0.33 \\
\hline $\mathrm{T} 3$ & -2.700 & 0.48 & -1.324 & 0.82 & -2908 & 0.60 & -0.761 & 0.86 & -1.267 & 0.80 & 1.765 & 0.73 \\
\hline CIS & 2.026 & 0.28 & 0.836 & 0.27 & 4.363 & 0.03 & 2.930 & 0.17 & 2.007 & 0.42 & 0.657 & 0.79 \\
\hline Unknown & 5.721 & 0.59 & $\mathrm{Na}$ & $\mathrm{Na}$ & -8.785 & 0.12 & -0.833 & 0.95 & -7.227 & 0.61 & -1.205 & 0.93 \\
\hline \multicolumn{13}{|l|}{$\mathrm{N}$ stage } \\
\hline No & ref & $\mathrm{Na}$ & ref & $\mathrm{Na}$ & ref & $\mathrm{Na}$ & ref & $\mathrm{Na}$ & ref & $\mathrm{Na}$ & ref & $\mathrm{Na}$ \\
\hline $\mathrm{N}+$ & 1.096 & 0.52 & -0.949 & 0.73 & 6.281 & 0.02 & 2.179 & 0.26 & 2.036 & 0.37 & -4.348 & 0.06 \\
\hline \multicolumn{13}{|c|}{ Systemic therapy } \\
\hline None & ref & $\mathrm{Na}$ & ref & $\mathrm{Na}$ & ref & $\mathrm{Na}$ & ref & $\mathrm{Na}$ & ref & $\mathrm{Na}$ & ref & $\mathrm{Na}$ \\
\hline CTx & -2.658 & 0.25 & -2.189 & 0.55 & 3.586 & 0.31 & -1.953 & 0.47 & -4.660 & 0.14 & 1.281 & 0.68 \\
\hline ETx & -4.394 & 0.03 & 1.402 & 0.66 & -2.850 & 0.36 & 0.201 & 0.93 & 4.366 & 0.11 & 2.341 & 0.39 \\
\hline CTx \& ETx & -0.246 & 0.90 & 1.433 & 0.65 & 2.442 & 0.42 & 0.053 & 0.98 & 3.199 & 0.23 & 2.638 & 0.32 \\
\hline \multicolumn{13}{|c|}{ Radiation therapy } \\
\hline $\begin{array}{l}\text { RTx } \\
\text { following } \\
\text { BCS }\end{array}$ & ref & $\mathrm{Na}$ & ref & $\mathrm{Na}$ & ref & $\mathrm{Na}$ & ref & $\mathrm{Na}$ & ref & $\mathrm{Na}$ & ref & $\mathrm{Na}$ \\
\hline No RTx & -1.693 & 0.62 & -1.144 & 0.83 & 4.617 & 0.36 & 5.290 & 0.17 & -3.956 & 0.38 & 11.956 & $0.009 *$ \\
\hline $\begin{array}{l}\text { Thoracic wall } \\
\text { and/or } \\
\text { locoregional } \\
\text { RTx }\end{array}$ & -5.323 & 0.13 & -2.702 & 0.63 & 2.890 & 0.58 & -3.017 & 0.46 & -4.903 & 0.30 & 8.860 & 0.07 \\
\hline \multicolumn{13}{|l|}{$B R C A$} \\
\hline $\begin{array}{r}\text { BRCA1/2 } \\
\text { negative }\end{array}$ & ref & $\mathrm{Na}$ & ref & $\mathrm{Na}$ & ref & $\mathrm{Na}$ & ref & $\mathrm{Na}$ & ref & $\mathrm{Na}$ & ref & $\mathrm{Na}$ \\
\hline $\begin{array}{l}\text { BRCA1/2 } \\
\text { positive }\end{array}$ & 4.602 & 0.04 & -5.429 & 0.13 & -1.371 & 0.69 & 1.454 & 0.58 & -3.997 & 0.19 & -5.242 & 0.09 \\
\hline Unknown & -0.437 & 0.78 & -2.408 & 0.34 & 1.653 & 0.49 & -2.311 & 0.20 & -4.516 & 0.03 & -0.969 & 0.65 \\
\hline
\end{tabular}

The multivariable linear regression model was composed of all baseline characteristics present in the left column of the table

$B C T$ breast-conserving therapy, MAS mastectomy, REC I mastectomy followed by (in)direct implant reconstruction, REC A mastectomy followed by (in)direct autologous reconstruction, $C T x$ chemotherapy and/or immunotherapy, ETx endocrine therapy, $R T x$ radiation therapy

*Significant beta coefficient

${ }^{\circ}$ Higher scores represent higher quality 
data could be used to build models to perform case-mix analyses which could be validated in other cohorts. The response rate for sexual functioning (EORTC-QLQ-BR23) was lower compared with other PROs, except for patients with breast reconstruction. Therefore, scores for sexual functioning might have been biased. Previous studies on sexual health in breast cancer patients showed that 50-90\% of women experience sexual dysfunction ${ }^{19,20}$ and that breast cancer surgery has a negative impact. ${ }^{21}$ The VBHC initiative, with questions regarding sexual functioning, could possibly open up the conversation and future consultation on sexuality in breast cancer patients at the outpatient clinic. Data on sexual functioning are hampered by the lower response rate and the lack of longitudinal data, limiting the clinical applicability of these scores.

There were no statistically significant differences in PROs between PBC and SBC patients. This conclusion is hampered concerning the BREAST-Q questionnaire. In the SBC group, in which patients are more often operated on both breasts, the applicability of the BREAST-Q is lower, since it does not account for two operated breasts or different types of breast surgery.

Measuring PROs during treatment has the potential to monitor and detect changes in physical or psychosocial problems at the outpatient clinic. Consequently, targeted supportive care concerning health-related QoL may be provided and possibly improve the care delivered. ${ }^{9,22}$ This evaluation enables a first insight into PRO scores according to patient, tumor, and treatment characteristics. Reference scores for the different PROs are pivotal when PROs are being used at the outpatient clinic to tailor and improve the care delivered. Knowledge on differences in satisfaction scores per type of breast cancer surgery performed can be used for shared decision-making. ${ }^{16}$ However, it should be stressed that we cannot determine a causal relation between the different treatments and outcome yet. Effects of treatments in observational data are potentially biased by confounding by indication and selection and should be interpreted with caution. Prospective and repeated evaluations of PROs throughout care form the cornerstone of VBHC and potentially enable more patient-centered breast cancer care with the possibility of improved shared treatment decision-making in breast cancer patients.

\section{CONCLUSIONS}

PROs were evaluated in 764 historical patients according to patient, tumor, and treatment characteristics in a single center. "Satisfaction with breasts" differed between type of surgery delivered. This knowledge as well as the collection of reference values could add value in shared decision-making concerning breast cancer surgery.
ACKNOWLEDGMENTS The authors would like to thank all the patients for their participation.

DISCLOSURE All authors declare no potential or actual conflicts of interest.

OPEN ACCESS This article is distributed under the terms of the Creative Commons Attribution 4.0 International License (http://crea tivecommons.org/licenses/by/4.0/), which permits unrestricted use, distribution, and reproduction in any medium, provided you give appropriate credit to the original author(s) and the source, provide a link to the Creative Commons license, and indicate if changes were made.

\section{REFERENCES}

1. Jemal A, Bray F, Center MM, Ferlay J, Ward E, Forman D. Global cancer statistics. CA Cancer J Clin. 2011;61(2):69-90.

2. van der Waal D, Verbeek AL, den Heeten GJ, Ripping TM, TjanHeijnen VC, Broeders MJ. Breast cancer diagnosis and death in the Netherlands: a changing burden. Eur J Public Health. 2015;25(2):320-4.

3. Saadatmand S, Bretveld R, Siesling S, Tilanus-Linthorst MM. Influence of tumour stage at breast cancer detection on survival in modern times: population based study in 173,797 patients. BMJ. 2015;351:h4901.

4. Lagendijk M, van Maaren MC, Saadatmand S, Strobbe LJA, Poortmans PMP, Koppert LB, et al. Breast conserving therapy and mastectomy revisited: breast cancer-specific survival and the influence of prognostic factors in 129,692 patients. Int J Cancer. 2017; 142:165-75.

5. Fisher B, Anderson S, Bryant J, Margolese RG, Deutsch M, Fisher ER, et al. Twenty-year follow-up of a randomized trial comparing total mastectomy, lumpectomy, and lumpectomy plus irradiation for the treatment of invasive breast cancer. $N$ Engl J Med. 2002;347(16):1233-41.

6. Litiere S, Werutsky G, Fentiman IS, Rutgers E, Christiaens MR, Van Limbergen E, et al. Breast conserving therapy versus mastectomy for stage I-II breast cancer: 20 year follow-up of the EORTC 10801 phase 3 randomised trial. Lancet Oncol. 2012;13(4):412-9.

7. Porter ME. What is value in health care? $N$ Engl $J$ Med. 2010;363(26):2477-81.

8. Ong WL, Schouwenburg MG, van Bommel AC, Stowell C, Allison KH, Benn KE, et al. A standard set of value-based patient-centered outcomes for breast cancer: the International Consortium for Health Outcomes Measurement (ICHOM) initiative. JAMA Oncol. 2016;3:677-85.

9. Velikova G, Booth L, Smith AB, Brown PM, Lynch P, Brown $\mathrm{JM}$, et al. Measuring quality of life in routine oncology practice improves communication and patient well-being: a randomized controlled trial. J Clin Oncol. 2004;22(4):714-24.

10. Sobin LH, Gospodarowicz M, Wittekind C. TNM classification of malignant tumors. New York: Wiley; 2009.

11. EuroQol G. EuroQol-a new facility for the measurement of health-related quality of life. Health Policy. 1990;16(3):199-208.

12. Aaronson NK, Ahmedzai S, Bergman B, Bullinger M, Cull A, Duez NJ, et al. The European Organization for Research and Treatment of Cancer QLQ-C30: a quality-of-life instrument for use in international clinical trials in oncology. J Natl Cancer Inst. 1993;85(5):365-76.

13. Sprangers MA, Groenvold M, Arraras JI, Franklin J, te Velde A, Muller M, et al. The European Organization for Research and Treatment of Cancer breast cancer-specific quality-of-life 
questionnaire module: first results from a three-country field study. J Clin Oncol. 1996;14(10):2756-68.

14. Pusic AL, Klassen AF, Scott AM, Klok JA, Cordeiro PG, Cano SJ. Development of a new patient-reported outcome measure for breast surgery: the BREAST-Q. Plast Reconstr Surg. 2009;124(2):345-53.

15. Aaronson NK, Ahmedzai S, Bregman B, Bullinger M, Cull A, Duez NJ, et al. The European Organization for Research and Treatment of Cancer QLQ-C30: a quality-of-life instrument for use in international clinical trials in oncology. $J$ Natl. Cancer Inst. 1993;85:365-76.

16. Atisha D, Rushing C, Samsa G, Locklear T, Cox C, Shelley Hwang E, et al. A national snapshot of satisfaction with breast cancer procedures. Ann Surg Oncol. 2015;22(2):361-9.

17. Kim MK, Kim T, Moon HG, Jin US, Kim K, Kim J, et al. Effect of cosmetic outcome on quality of life after breast cancer surgery. Eur J Surg Oncol. 2015;41(3):426-32.

18. Howes BH, Watson DI, Xu C, Fosh B, Canepa M, Dean NR. Quality of life following total mastectomy with and without reconstruction versus breast-conserving surgery for breast cancer: a case-controlled cohort study. J Plast Reconstr Aesthetic Surg. 2016;69(9):1184-91.

19. Dizon DS. Quality of life after breast cancer: survivorship and sexuality. Breast J. 2009;15(5):500-4.

20. Thors CL, Broeckel JA, Jacobsen PB. Sexual functioning in breast cancer survivors. Cancer Control. 2001;8(5):442-8.

21. Cornell LF, Mussallem DM, Gibson TC, Diehl NN, Bagaria SP, McLaughlin SA. Trends in sexual function after breast cancer surgery. Ann Surg Oncol. 2017;24(9):2526-38.

22. Kotronoulas G, Kearney N, Maguire R, Harrow A, Di Domenico $\mathrm{D}$, Croy S, et al. What is the value of the routine use of patientreported outcome measures toward improvement of patient outcomes, processes of care, and health service outcomes in cancer care? A systematic review of controlled trials. J Clin Oncol. 2014;32(14):1480-501. 